\title{
(6) OPEN ACCESS \\ Distal access using hyperflexible atraumatic distal tip with optimized proximal stability of the Benchmark intracranial guide catheter for the treatment of cerebral vascular diseases: a technical note
}

\author{
Arun Chandran, ${ }^{1}$ Mani Puthuran, ${ }^{1}$ Paul R Eldridge, ${ }^{2}$ Hans C Nahser ${ }^{1}$
}

${ }^{1}$ Neuroradiology Department, The Walton Centre NHS Foundation Trust, Liverpool, UK ${ }^{2}$ Neurosurgery Department, The Walton Centre NHS Foundation Trust, Liverpool, UK

\section{Correspondence to}

Dr Arun Chandran,

Neuroradiology Department,

The Walton Centre, Lower

Lane, Fazakerley, Liverpool L9

7 L, UK; Arun.Chandran@

thewaltoncentre.nhs.uk

Received 12 April 2015 Revised 22 May 2015

Accepted 26 May 2015 Published Online First

12 June 2015

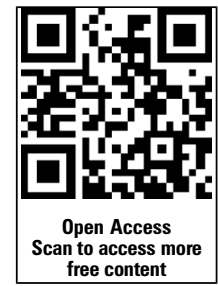

CrossMark

To cite: Chandran $\mathrm{A}$, Puthuran $\mathrm{M}$, Eldridge $\mathrm{PR}$ et al. I Neurolntervent Surg 2016;8:718-721.

\section{ABSTRACT}

Background A stable guide catheter position within the intracranial vasculature is critical for safe, successful endovascular treatment.

Objective To present ourinitial experience with the 0.071 inch inner diameter Benchmark guide catheter used in the treatment of intracranial cerebrovascular pathologies, demonstrating its safety and efficacy. Methods We retrospectively reviewed use of the Benchmark guide catheter from September through December 2014 in the management of various neuroendovascular intracranial pathologies. Clinical performance and complication rates were evaluated, with particular consideration of vessel tortuosity. A total of 62 Benchmarks were used, 47 in the anterior circulation, 10 in the posterior circulation, 4 in the external carotid, and 1 in the venous sinus. The five cases with access to the external carotid and venous sinus were excluded.

Results The Benchmark was able to cross at least one $90^{\circ}$ turn in $49(86 \%)$ of the 57 patients. Reversal of the catheter was seen in $15 \%$ of 47 anterior circulation cases (4 at one $90^{\circ}$ turn; 3 at two $90^{\circ}$ turns). We report no complications of dissection or thromboembolic events. All guide catheter positions were safely achieved over a 0.035 Terumo stiff glidewire without need for an inner smaller lumen guide catheter for navigation.

Conclusions Benchmark is a new guide catheter, with an ideal combination of both hyperflexible, atraumatic distal tip and optimized proximal shaft support to provide stable $6 \mathrm{~F}$ primary access for a successful neurointerventional procedure. Benchmark can be easily, safely, and consistently positioned in a desired location within intracranial arteries providing a stable position for intervention and adequate angiography.

\section{INTRODUCTION}

The field of neurointerventional radiology is constantly advancing, especially over the past two decades. New technology deriving from advances in material sciences is constantly being developed, and tried and tested in this field to improve patient care and safety in the delivery of minimally invasive treatments for cerebrovascular pathologies. ${ }^{1}{ }^{2}$ The fundamental of a successful endovascular intervention is a safe guide catheter position placed as close to the target of treatment as possible in a safe and efficient manner. The devices-coils, stents, balloons, and so forth-can then be used to treat the pathology. The ability to treat increasing spectrum pathologies is partly dependent on the successful positioning of such a device. Despite improvement of the materials and catheters used, the extra- and intracranial tortuous vasculature poses challenges in the treatment of cerebrovascular pathologies. Various alternative methods have been used to overcome this problem by direct carotid and vertebral artery puncture, radial/brachial access, and adaptation of catheter techniques such as buddy wire, coaxial delivery of guide catheters. ${ }^{3-9}$

In a recent review by Turk et $a l,{ }^{10}$ the safety and efficacy of the newer generation 0.070 guide catheter was demonstrated with high success and a low complication rate while obtaining intracranial access. In the above-mentioned review, Neuron (Penumbra Inc, Oakland, California, USA) 0.070 guide catheter performance testing was part of the study proving safety and efficacy.

With further recent advancement, especially in aneurysm embolization using flow diversion and flow disruption, there is a need for a stable construct to deliver these devices owing to their material stiffness. At the same time, adequate angiographic visualization of the target lesion is also necessary to plan and perform safe treatment. This warrants a $6 \mathrm{~F}$ guide catheter that can provide stable, safe, and consistent distal intracranial access.

In our study, we aim to assess the safety and efficacy of a newer generation Benchmark (Penumbra Inc) 0.071 inch inner diameter guide catheter used in the treatment of intracranial cerebrovascular pathologies. This catheter is an improved version of Neuron 0.070, with an ideal combination of both hyperflexible and atraumatic distal tip yet ultrasupportive proximal shaft that provides enhanced trackability and stability.

\section{METHODS}

A retrospective review was carried out of sequential consecutive use of the Benchmark guide catheter in the 4-month period of September 2014 through December 2014, in the therapeutic management of various neuroendovascular intracranial pathologies.

This study was approved by the local institutional review board. We excluded those cases where guide catheter stability was not needed, such as extracranial interventions including external carotid access, and cervical carotid and vertebral artery interventions. 
Figure 1 Lateral view of $(A)$ internal carotid artery and (B) anteroposterior view of vertebral artery demonstrating the scoring system used for assigning the clinical performance of the Benchmark catheter over $90^{\circ}$ turns.
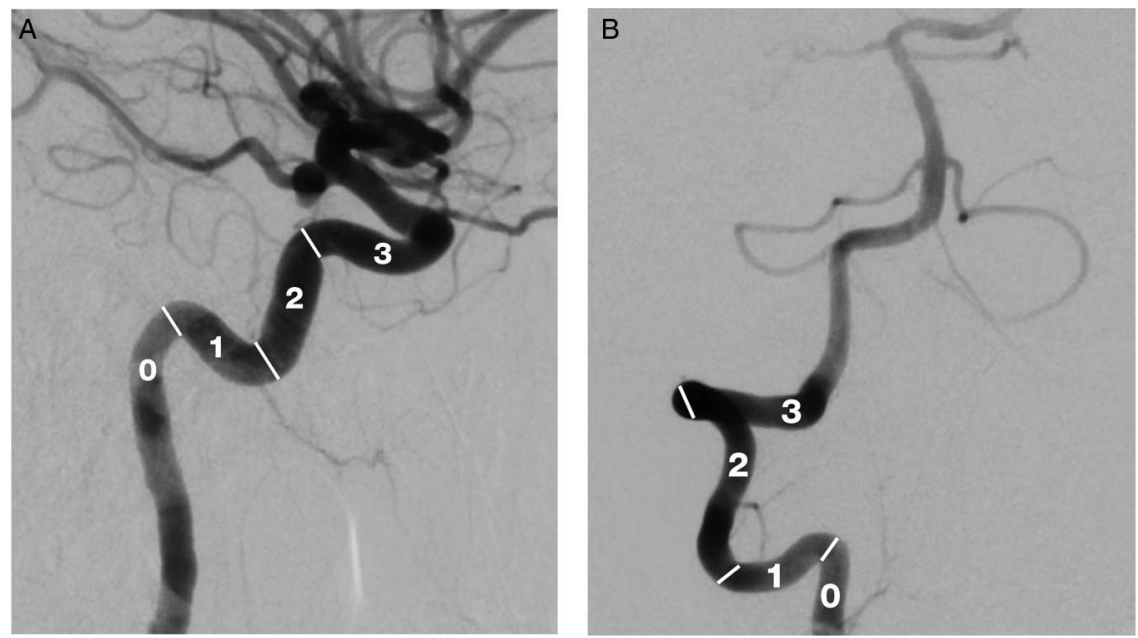

Clinical performance or success of guide catheter placement was measured by the number of acute right angle $\left(90^{\circ}\right)$ turns the catheter could achieve safely, especially in the anterior and posterior circulation (figure 1). A score of 1 is given for each curve negotiated; an acute $90^{\circ}$ curve in the cervical carotid is given a score of 1 (figure $2 \mathrm{~A}, \mathrm{~B}$ ). A score of 0 is given if the guide catheter is placed in a straight cervical carotid or V2 segment of the vertebral artery.

A failure in clinical performance of the guide catheter was measured by the reversal (back out by one $90^{\circ}$ turn) of access during the procedure by recording the final guide catheter position (figure 2C, D). The guide catheter-related complications were only considered if they were directly related to placement and delivery of the catheter during access. The technique used to guide the Benchmark into the landing position was also recorded.

\section{RESULTS}

Sixty-two Benchmark guide catheters were used during the study: 47 in the anterior circulation, 10 in the posterior circulation, 4 in the external carotid, and 1 in the venous sinus. Five cases with access to the external carotid artery and venous sinus
Figure 2 ( $A$ and $B$ ) Anteroposterior and lateral view of the internal carotid artery (ICA) illustrating cervical $90^{\circ}$ turns and how the scoring system is adapted to assess clinical performance of the Benchmark catheter. If the guide catheter lands in the cervical ICA, a score of 2 is given for crossing two acute $90^{\circ}$ curves in the cervical carotid. (C and D) The reversal of access was judged on the back out of the Benchmark catheter at the end of the procedure. In this example the initial score was 2 with the access landing zone (white thin arrow) in the vertical petrous ICA. The final position of the guide catheter position (white thick arrow) was in the cervical ICA. The solid arrowhead represents the proximal coil detachment marker during the final stages of treatment. The reversal score would be -2 owing to back out by two $90^{\circ}$ curves.
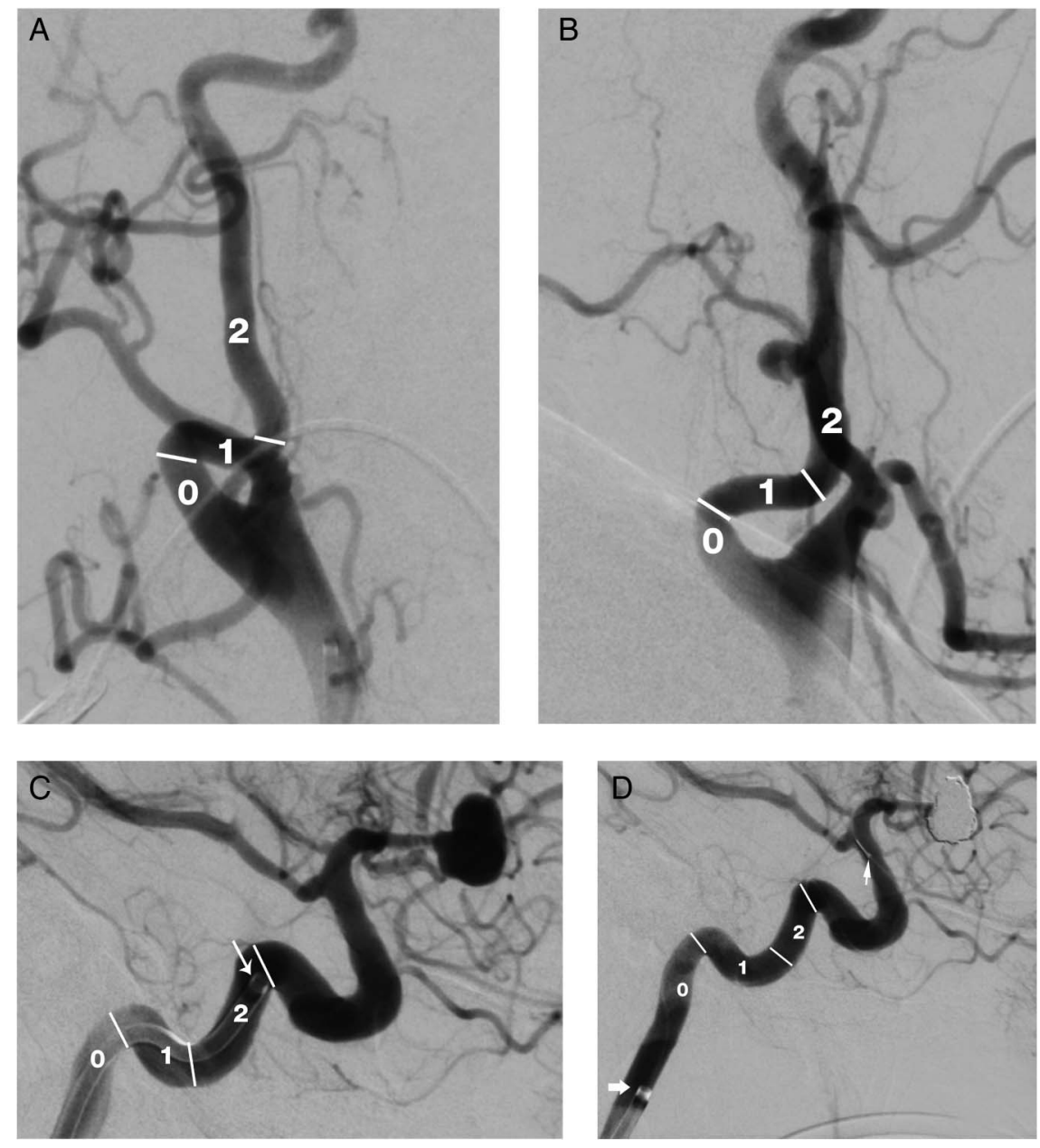
Table 1 Number of $90^{\circ}$ turns negotiated by Benchmark

\begin{tabular}{lllrrr}
\hline Cases & No & Score-0 & Score-1 & Score-2 & Score-3 \\
\hline ICA & 47 & $6(12.8)$ & $9(19.1)$ & $23(48.9)$ & $9(19.1)$ \\
Vertebral & 10 & $2(20.0)$ & $2(20.0)$ & $3(30.0)$ & $3(30.0)$ \\
Total & 57 & $8(14.0)$ & $11(19.3)$ & $26(45.6)$ & $12(21.1)$ \\
\hline
\end{tabular}

Results are shown as number (\%).

ICA, internal carotid artery.

were excluded as it was deemed that guide catheter stability would not affect the success of the procedure. Thus 57 subjects were included in the study.

The procedures included treatments of 54 aneurysms, of which 45 were primary bare coil embolizations, 3 stent-assisted coiling, 2 WEB embolizations, 2 primary intracranial stenting, 1 pulse rider-assisted coiling, and 1 flow diverter treatment. The remaining three were arteriovenous malformation embolisations: two involved anterior and one involved posterior circulation.

When the target pathology was in the anterior circulation, the Benchmark was able to cross at least one $90^{\circ}$ turn in $87 \%$ of cases, and an intracranial access (horizontal petrous internal carotid artery (ICA) and above) was obtained in $85 \%$ of the cases (tables 1 and 2). In total of 47 cases of anterior circulation access, successful intracranial guide catheter placements were included in 9 cases in the horizontal petrous segment, 23 cases in the vertical petrous segment, and 8 cases in the cavernous petrous segment. Reversal of access of $15 \%$ (four cases by one $90^{\circ}$ turn and three cases by two $90^{\circ}$ turns) was seen in the anterior circulation. The vertical petrous and cavernous segment placement accounted for $86 \%$ and $14 \%$ chances of guide catheter kickback during the procedure.

When the target pathology was in the posterior circulation in a total of 10 cases, the Benchmark was able to cross at least one $90^{\circ}$ turn in $80 \%$ of cases (table 2). The levels of access were as follows: V2 segment (20\%), proximal (20\%), mid (30\%), and distal (30\%) access in the vertebral V3 segment. There was no reversal of access in the posterior circulation.

The Benchmark provided support to complete the intervention in $100 \%$ of cases, regardless of the final location of the catheter tip. Overall, even after reversal of access during a procedure, the Benchmark was able to provide intracranial access in $85 \%$ of cases, especially in the anterior circulation. We report no complications of dissection or thromboembolic events related to the Benchmark catheter in our current series.

We used a diagnostic catheter to perform an initial angiogram and plan intervention. All our guide catheter positions were safely achieved over a 0.035 Terumo glidewire without the need for an inner small guide catheter for negotiation. The positioning of the Benchmark guide catheter in its target location is achieved by an exchange maneuver over a long stiff 0.035 Terumo glide wire across the aortic arch. This technique in our experience is safe and cost-effective.

There were two cases of cervical ICA acute $90^{\circ}$ loops in our series; a representative example is shown in figure 2.

\section{DISCUSSION}

In our report we describe our experience with the newer generation $6 \mathrm{~F}$ or 0.071 inner diameter Benchmark guide catheter for distal intracranial access in therapeutic neuroendovascular treatments. A successful and safe neuroendovascular treatment is based on the fundamentals of a stable and distal (close to target) position of the guide catheter. Traditional or conventional guide catheters relied on catheter stiffness to provide stability, thus inherently jeopardizing the navigability. ${ }^{1}$

The current generation guide catheter technology is based on varying lengths of material and stiffness in the construct to provide optimal stability with a suitable navigability for safety in the intracranial circulation. These recent technological advances have overcome the alternative methods of catheter stability and access using direct cervical carotid puncture, radial artery access, and other catheter-related techniques such as buddy wire. ${ }^{3-9}$

The traditional aim of guide catheter positioning in the intracranial vasculature is twofold. First, is to gain access close to the target pathology for safe one-to-one manipulation of microwire and catheters. Second, is to prevent proximal migration of the guide catheter due to tension and redundancy created within the tortuous cranial vasculature with resultant loss of microcatheter position.

The Benchmark guide catheter deals with both these concerns, delivering next-generation guide catheter performance through optimized support and stability. The atraumatic hyperflexible distal tip of the Benchmark allows access as close to the target as needed. The optimized proximal shaft stiffness provides two features: first, it provides enhanced support at the aortic arch, even in cases with difficult anatomy; second, the translation of the movement to the distal tip while gaining access is smooth and close to a 1:1 ratio, leading to optimized smooth navigability. The lack of the latter feature is mostly the cause of dissection while gaining access in previous generation guide catheters.

The most recent and largest review on current generation guide catheter safety and efficacy was published by Turk et al, ${ }^{10}$ where the performance of the Neuron 0.070 catheters was illustrated. The Benchmark 0.071 inner diameter distal guide

Table 2 Landing and final position to illustrate success and failure in clinical performance

\begin{tabular}{llccc}
\hline $\mathbf{4 7}$ Cases & Cervical ICA & H-petrous & V-petrous & Cavernous \\
\hline $\begin{array}{l}\text { Landing position } \\
\text { Final position }\end{array}$ & $7(14.9)$ & $9(19.1)$ & $23(48.9)$ & $17(36.2)$ \\
& $9(19.1)$ & $14(29.8)$ & & $7(17.0)$ \\
\hline 10 cases & V2 segment & Prox-V3 & Mid-V3 & Distal-V3 \\
\hline $\begin{array}{l}\text { Posterior circulation } \\
\text { Final position }\end{array}$ & $2(20.0)$ & $2(20.0)$ & $3(30.0)$ & $3(30.0)$ \\
\hline Results are shown as number (\%). & $2(20.0)$ & $2(20.0)$ & $3(30.0)$ & $3(30.0)$ \\
ICA, internal carotid artery. & & &
\end{tabular}


catheter for intracranial access is an improvement in technology over the 0.070 Neuron. The Benchmark catheter provides a hyperflexible distal tip and improved proximal support for stable, safe, and consistent intracranial access. In our series we were able to show that the tracking and arch support features of the Benchmark enabled successful completion of all interventions, regardless of final guide catheter position.

These results may even underestimate the performance owing to factors such as variance of the threshold of catheter tip landing zone among operators. In our experience, an understanding of the material and technique plays an important role in establishing stable and safe guide catheter tip position.

The practice in our center is to guide the Benchmark over a stiff Terumo 0.035 glidewire, which in our hands has provided safe delivery of the catheter to its landing. This is also cost-effective in comparison with a coaxial technique. The previous generation 0.070 Neuron in our experience requires catheter hub manipulation to navigate around the sharp carotid siphons. The new Benchmark catheter tracks and navigates with ease over a 0.035 Terumo glidewire at the sharp angles owing to its hyperflexible and atraumatic distal tip. To an extent, Benchmark even bypasses the learning curve among practitioners in transition between use of older and newer generation guide catheters. However, careful and safe cautious delivery of the catheter is always recommended.

In our series there was an increased chance of reversal if the landing zone was within the vertical petrous ICA. This might be explained by the fact that the most common landing zone was the vertical petrous in our series, at close to $50 \%$ of cases. However, we would still recommend the vertical petrous ICA as the most desirable position for guide catheter landing in our practice. This landing zone position will allow stable access even after reversal into the horizontal petrous ICA. Also, the guide catheter can be negotiated over the microcatheter, if necessary, to reclaim the landing zone for stability if safe to do so.

The limitations of the study for comparisons with other devices are its retrospective design and its modest sample size. However, such a retrospective study can provide useful information about safety of the device-these data do demonstrate the inherent safety of the device. As previous case series and comparative studies ${ }^{10-12}$ have used conventional rigid catheters and newer generation guide catheters, the authors felt there was only a need to establish the safety and efficacy of this newer device. This Benchmark device is an improved version of the previous generation $6 \mathrm{~F}$ Neuron, which has an established safety and efficacy record among practitioners in our study.

\section{CONCLUSION}

Our study represents clinical experience in a tertiary neurosciences center with a high volume neuroendovascular practice, where Benchmark is the newest guide catheter to be adopted as a primary access device. In our experience this catheter has demonstrated high standards of safety and clinical efficacy. Benchmark can be easily, safely, and consistently positioned in a desired location within the intracranial arteries over a 0.035 Terumo glidewire that provides a stable position for intervention and adequate angiography.

Contributors $\mathrm{AC}$ wrote and reviewed the manuscript, designed and conducted the study, and analyzed the data. MP and PRE reviewed the manuscript. HCN designed the study and reviewed the manuscript.

Competing interests None declared.

Ethics approval Institutional review board approval obtained from The Walton Centre NHS Foundation Trust.

Provenance and peer review Not commissioned; externally peer reviewed.

Open Access This is an Open Access article distributed in accordance with the Creative Commons Attribution Non Commercial (CC BY-NC 4.0) license, which permits others to distribute, remix, adapt, build upon this work non-commercially, and license their derivative works on different terms, provided the original work is properly cited and the use is non-commercial. See: http://creativecommons.org/ licenses/by-nc/4.0/

\section{REFERENCES}

1 Linfante I, Wakhloo AK. Brain aneurysms and arteriovenous malformations: advancements and emerging treatments in endovascular embolization. Stroke 2007;38:1411-17.

2 Boulos AS, Levy El, Bendok BR, et al. Evolution of neuroendovascular intervention: a review of advancement in device technology. Neurosurgery 2004;54:438-52.

3 Blanc R, Piotin M, Mounayer $C$, et al. Direct cervical arterial access for intracranial endovascular treatment. Neuroradiology 2006;48:925-9.

4 Nii K, Kazekawa K, Onizuka M, et al. Direct carotid puncture for the endovascular treatment of anterior circulation aneurysms. AJNR Am J Neuroradiol 2006;27:1502-4

5 Yuzawa I, Kurata A, Suzuki S, et al. Efficacy of a direct puncture approach for anterior circulation aneurysms using a newly developed guiding catheter-especially for geriatric patients. Surg Neurol 2007;67:30-4.

6 Simon SD, Ulm AJ, Russo A, et al. Distal intracranial catheterization of patients with tortuous vascular anatomy using a new hybrid guide catheter. Surg Neurol 2009;72:737-40

7 Lee $\mathrm{TH}$, Choi $\mathrm{CH}$, Park KP, et al. Techniques for intracranial stent navigation in patients with tortuous vessels. AJNR Am J Neuroradiol 2005;26:1375-80.

8 White JB, Kallmes DF. Utility of the "buddy" wire in intracranial procedures. Neuroradiology 2008;50:185-7.

9 Bendok BR, Przybylo JH, Parkinson R, et al. Neuroendovascular interventions for intracranial posterior circulation disease via the transradial approach: technical case report. Neurosurgery 2005;56:E626.

10 Turk A, Manzoor MU, Nyberg EM, et al. Initial experience with distal guide catheter placement in the treatment of cerebrovascular disease: clinical safety and efficacy. J Neurointerv Surg 2013:5:247-52.

11 Chaudhary N, Pandey AS, Thompson BG, et al. Utilization of the Neuron 6 French 0.053 inch inner luminal diameter guide catheter for treatment of cerebral vascular pathology: continued experience with ultra distal access into the cerebral vasculature. J Neurointerv Surg 2012;4:301-6.

12 Lin LM, Colby GP, Huang J, et al. Ultra-distal large-bore intracranial access using the hyperflexible Navien distal intracranial catheter for treatment of cerebrovascular pathologies: a technical note. J Neurointerv Surg 2014;6:301-7. 